\title{
An Unexpected Complication in a Pediatric Patient With Sclerosing Cholangitis: A Huge Spontaneous Biloma
}

\author{
Aylin Yucel ${ }^{1}$, Hatice Mutlu Albayrak², Meltem Gumus ${ }^{3}$, Mehmet Asil ${ }^{4}$, Orhan Ozbek ${ }^{5}$, Hasan Ali Yuksekkaya ${ }^{1}$ \\ ${ }^{1}$ Department of PediatricGastroenterology, HepatologyandNutrition, Meram MedicalFaculty, Necmettin Erbakan University, Konya, Turkey \\ ${ }^{2}$ Department of Pediatric Genetics, Ondokuz Mayıs UniversityMedicalFaculty, Samsun, Turkey \\ ${ }^{3}$ Department of PediatricGastroenterology, HepatologyandNutrition, Konya Training andResearchHospital, Konya, Turkey \\ ${ }^{4}$ Department of GastroenterologyandHepatology, Meram MedicalFaculty, Necmettin Erbakan University, Konya, Turkey \\ ${ }^{5}$ Department of Radiology, Meram MedicalFaculty, Necmettin Erbakan University, Konya, Turkey
}

\begin{abstract}
Introduction: Biloma is encapsulated collection of bile due to iatrogenic injury or abdominal trauma. The perforation of the extra-intrahepatic bile duct without any traumatic or iatrogenic injury is defined as spontaneous biloma. The spontaneous biloma is very rare and infrequently reachesto huge sizes. Previously described causes of spontaneous biloma include choledocholithiasis, acute cholecystitis, cholangiocarcinoma, pancreatic malignancy and hepatic abscesses or infarctions.

Case Presentation: We describe the case of a huge spontaneous biloma in a 16-year-old girl patient with sclerosing cholangitis. She applied to our hospital for generalised abdominal distention and pain. There was no cholestasis at her admission, but, interestingly cholestasis developed on the tenth day of hospitalization.She was treated with percutaneous drainage, endoscopic sphincterotomy and stent placement into the common bile duct. After discharge, there was no recurrence at the three-month follow-up.

Conclusions: To our knowledge, this is the first report in the literature of spontaneous biloma associated with sclerosing cholangitis.
\end{abstract}

\section{Introduction}

Biloma is a rare disease defined as an encapsulated collection of bile due to disruption of the biliary tree [1]. The majority of bile leaks occur secondary to traumatic or iatrogenic injury, including abdominal surgery, laparoscopic surgery, and percutaneous catheter drainage. Spontaneous perforation of the biliary tree without anprevious history of trauma or surgery is an extremely rare cause of biloma [2].

The pathophysiology of spontaneous biloma is not fully clear, but one suggested contributing factor is increased intraductal pressure due to obstructive lesions or infarctions in any part of the biliary tree. Most cases of previously reported spontaneous bilomas occurred secondary to choledocholithiasis or cholangiocarcinoma. Other rare causes described in the literature are acute cholecystitis, pancreatic adenocarcinoma, sickle cell disease and tuberculosis [3].

The complications of sclerosing cholangitis are recurrent cholangitis, cirrhosis, cholangiocarcinoma, hepatocellular carcinoma, gallbladder carcinoma, cholelithiasis, fat-soluble vitamin deficiencies and osteoporosis. Additionally, primary sclerosing cholangitis has be enassociatedwithinflammatoryboweldisease [4]. Spontaneous biloma formation with sclerosing cholangitis has not been reported before, according to our best knowledge. Here, we reported a patient with huge spontaneous biloma-related sclerosing cholangitis.

\section{Case Presentation}

A 16-year-old female patient was admitted to our hospital with nausea, generalized abdominal pain and distention without fever or jaundice. It was learnt that she got a diagnosisof celiac disease twelve years ago, and sclerosing cholangitis four years ago.The lower gastrointestinal endoscopy and histopathology carried out to exclude inflammatory bowel disease were normal. She was taking

\section{Publication History:}

Received: February 15, 2016

Accepted: May 16, 2016

Published: May 18, 2016

\section{Keywords:}

Status epilepticus, Neuronal damage, Classification, Treatment prednisolone $(5 \mathrm{mg} /$ day $)$ and azathioprine $(50 \mathrm{mg} /$ day $)$ for four years. There was no history of trauma and/or abdominal surgery. Four months ago, magnetic resonance imaging (MRI) and magnetic resonance cholangiopancreatography (MRCP) revealed distal narrowing and proximal dilatation of the common hepatic duct (CHD) and dilated intrahepatic bile ducts (IBDs). In addition, the dilatation of the left intrahepatic bile ducts was more pronounced than the right intrahepatic bile ducts (Figure 1). On physical examination, her weight was $32 \mathrm{~kg}$, height was $145 \mathrm{~cm}$ and body massindexwas 15,2 (all of them below the 3rd percentile for her age). Her pubertaldevelopmentwas at Tanner stage 5. Thepatient's body temperaturewas $36,8{ }^{\circ} \mathrm{C}$, herbloodpressurewas $110 / 60 \mathrm{~mm} /$ $\mathrm{Hg}$ and her heart rate was 88 beatsperminute. She had distinct abdominal distention. Abdominal percussion revealed dull sounds throughout the right upper and lower quadrants. Complete blood count revealed microcytic anaemia (haemoglobin $10.6 \mathrm{~g} / \mathrm{dL}$, mean corpuscular volume $75.2 \mathrm{fL}$ ). Erythrocyte sedimentation rate (ESR) $=63 \mathrm{~mm} / \mathrm{h}$, and C-reactive protein $(\mathrm{CRP})=12.9 \mathrm{mg} / \mathrm{L}($ range $0-5$ $\mathrm{mg} / \mathrm{L}), A S T=43 \mathrm{IU} / \mathrm{L}, \mathrm{ALT}=32 \mathrm{IU} / \mathrm{L}$, total bilirubin $=0.74 \mathrm{mg} / \mathrm{dl}$, direct bilirubin $=0.34 \mathrm{mg} / \mathrm{dl}$, alkaline phosphatase $=116 \mathrm{IU} / \mathrm{L}$, gammaglutamyltranspeptidase $(\mathrm{GGT})=38 \mathrm{IU} / \mathrm{L}, \mathrm{CA} 19-9=32 \mathrm{IU} / \mathrm{mL}(0-36$ $\mathrm{IU} / \mathrm{mL}$ ), and carcinoembryonic antigen $(\mathrm{CEA})=2.6 \mathrm{ng} / \mathrm{ml}$. Her renal function tests and electrolytes levels were normal.

"Corresponding Author: Dr. Aylin Yücel, Necmettin Erbakan University, Meram Medical Faculty, Department of Pediatrics, Division of Pediatric Gastroenterology Hepatology and Nutrition, 42080 Meram, Konya, Turkey, Tel: +90 332223 7415; E-mail: ayucel82@hotmail.com

Citation: Yucel A, Albayrak HM, Gumus M, Asil M, Ozbek O,A, et al. (2016) An Unexpected Complication in a Pediatric Patient With Sclerosing Cholangitis: A Huge Spontaneous Biloma. Int J Pediatr Neonat Care 2: 116. doi: https://doi. org/10.15344/2455-2364/2016/116

Copyright: () 2016 Yucel et al. This is an open-access article distributed under the terms of the Creative Commons Attribution License, which permits unrestricted use, distribution, and reproduction in any medium, provided the original author and source are credited. 
Citation: Yucel A, Albayrak HM, Gumus M, Asil M, Ozbek O,A, et al. (2016) An Unexpected Complication in a Pediatric Patient With Sclerosing Cholangitis: A Huge Spontaneous Biloma. Int J Pediatr Neonat Care 2: 116. doi: https://doi.org/10.15344/2455-2364/2016/116

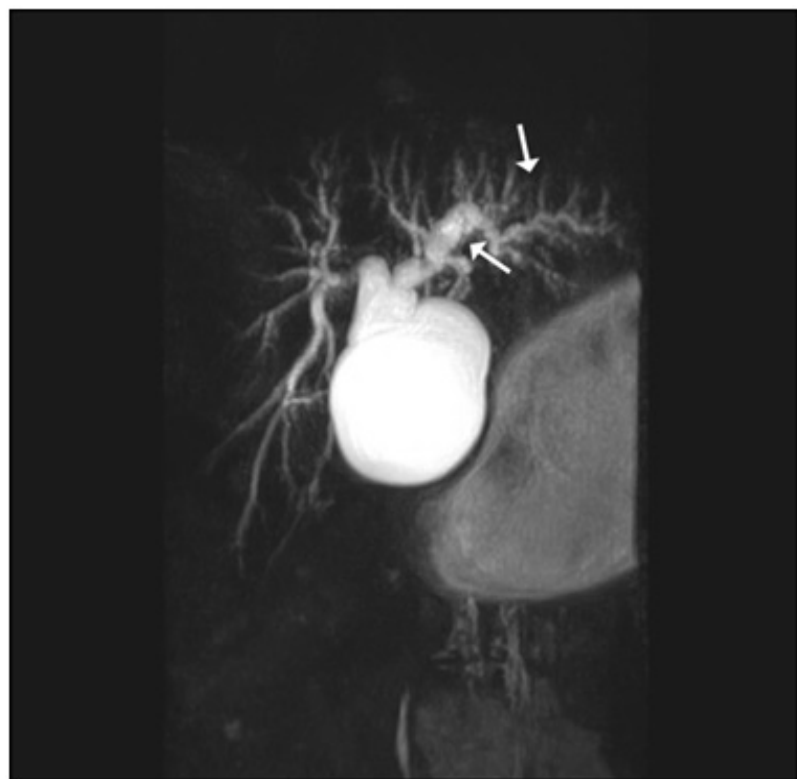

Figure 1: MRCP images obtained four months prior to biloma formation. The intrahepatic bile ducts appear wide (pruned-tree appearance).

Ductal dilatation is more pronounced in the left intrahepatic bile ducts.

Abdominal ultrasound and MRI demonstrated a loculated fluid collection extending from the subcapsular region of the right hepatic lobe to the pelvic inlet, measuring approximately $25 \times 12 \times 14 \mathrm{~cm}$ (Figure 2). However, no stones were observed in the gallbladder or biliary tree, nor in the mass. MRCP showed prominent dilatation of the proximal common hepatic bile duct and the left intrahepatic bile duct, and a slightly dilated right intrahepatic bile duct. Additionally, the distal part of the common bile duct (CBD) could not be visualized (Figure 3A-3B).

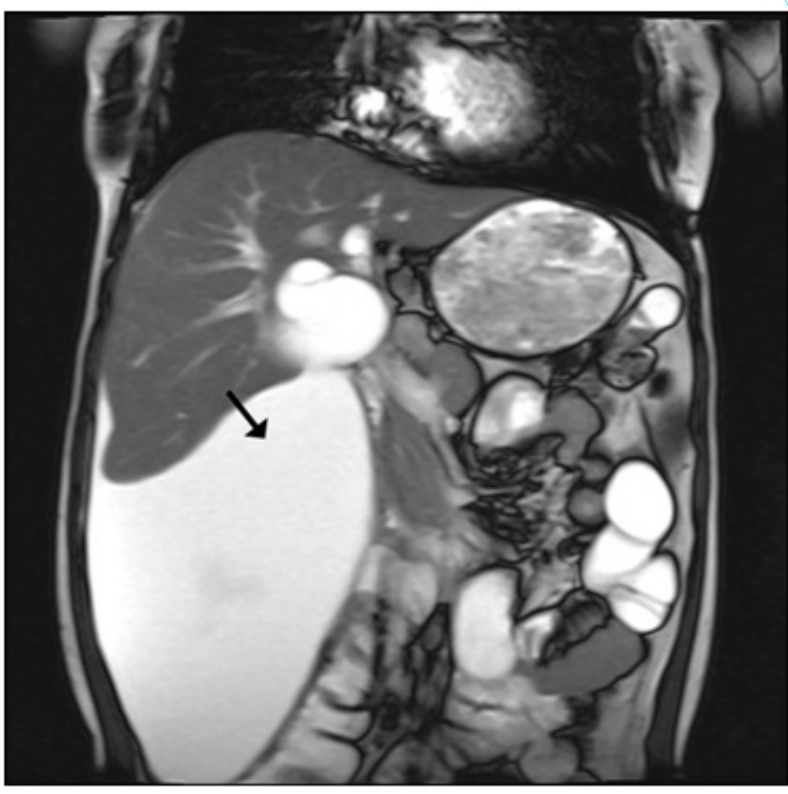

Figure 2: Abdominal MRI. There is a loculated fluid collection extending from the subcapsular region of the right hepatic lobe to the pelvic inlet, measuring approximately $25 \times 12 \times 14 \mathrm{~cm}$.

The loculated fluid was drained percutaneously by needle aspiration. The aspirated fluid total bilirubin level $=20.8 \mathrm{mg} /$ $\mathrm{dL}$ and a direct bilirubin level $=6.7 \mathrm{mg} / \mathrm{dL}$. Hence, the fluid was confirmed to be a biloma. No microorganism was detected on Gram staining. Culture and cytology of the aspirated fluid were negative. However, the same amount of liquid, by a percutaneous catheter (10-French), was reaccumulated within four days. Antibiotic treatment was initiated in order to prevent infection. Feeding was stopped and total parenteral nutrition was started to reduce bile

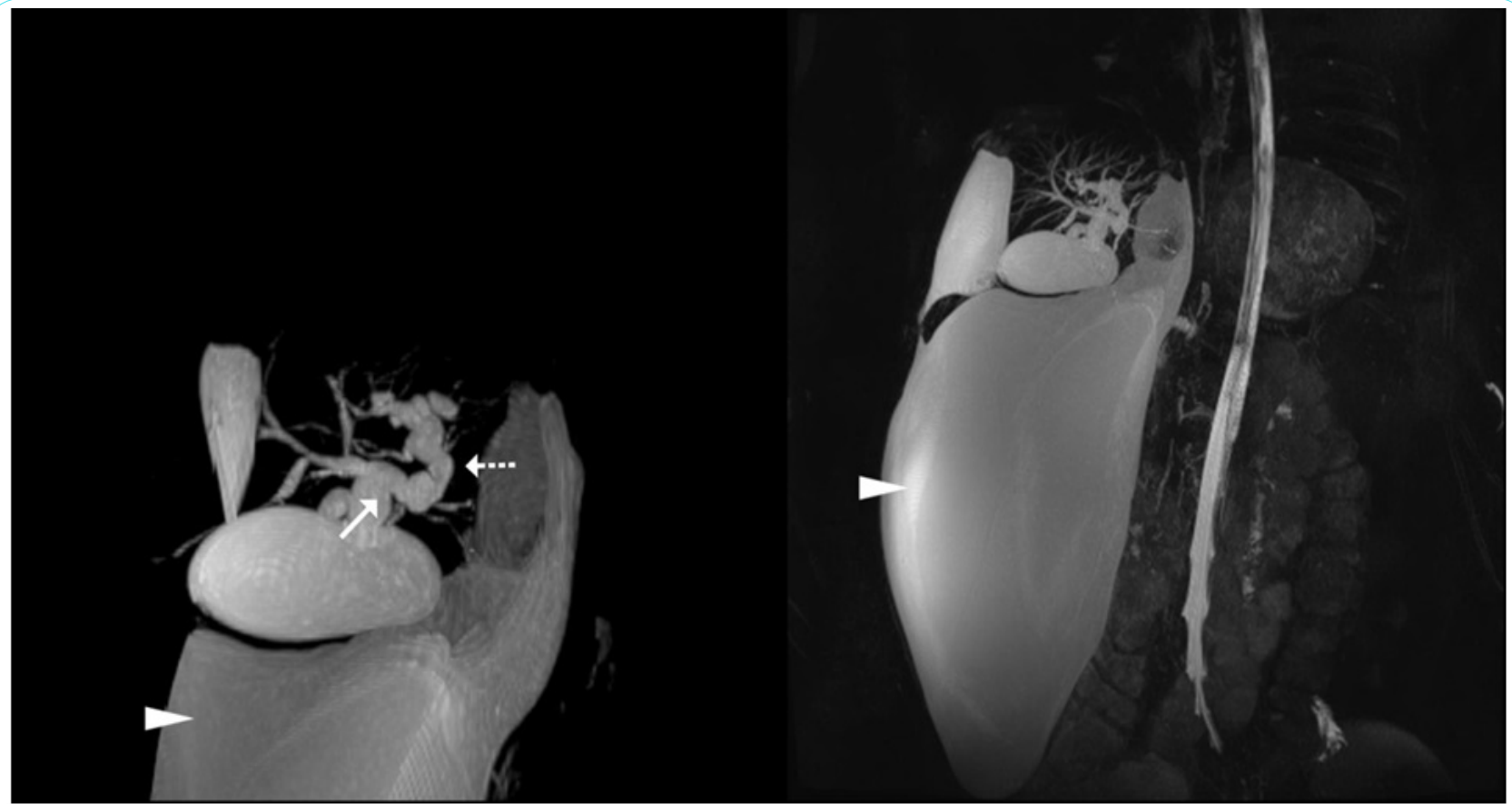

Figure 3: MRCP images. 3A: The proximal part of the common hepatic duct (arrow) and intrahepatic bile ducts are dilated. Multifocal strictures, dilatations and irregularity are seen, particularly in the left intrahepatic bile ducts (stippled arrow). There is a fluid collection suggesting biloma in the subhepatic region (arrowhead). 3B: MRCP image showing a huge loculated fluid collection extending from the subcapsular region of the right hepatic lobe to the pelvic inlet (arrowhead). 
Citation: Yucel A, Albayrak HM, Gumus M, Asil M, Ozbek O,A, et al. (2016) An Unexpected Complication in a Pediatric Patient With Sclerosing Cholangitis: A Huge Spontaneous Biloma. Int J Pediatr Neonat Care 2: 116. doi: https://doi.org/10.15344/2455-2364/2016/116

Page 3 of 5

production. Even then, approximately $10-20 \mathrm{ml}$ of liquid continued to flow from the drainage daily. Cholestasis developed on the tenth day of hospitalization (ALP=153 IU/L, GGT=398 IU/L, total bilirubin $5.72 \mathrm{mg} / \mathrm{dl}$, and direct bilirubin $4.07 \mathrm{mg} / \mathrm{dl}$ ). It was considered that the huge biloma could be compressing the $\mathrm{CBD}$ and than endoscopic retrograde cholangio-pancreatography (ERCP) was performed. ERCP showed compression of the CBD by a huge biloma. During the ERCP, we carried out an endoscopic sphincterotomy and a $10 \mathrm{Fr}$ stent was placed into the $\mathrm{CBD}$, to provide the decompression of $\mathrm{CBD}$ and reduce the intraductal pressure (Figure 4A-4B). Serum ALP, GGT and bilirubin levels subsequently decreased to normal levels, and the bile drainage ceased after two days. The percutaneous drainage catheter was removed, and the patient was discharged with full recovery. No recurrence-related clinic encountered during her 3 months of followup.

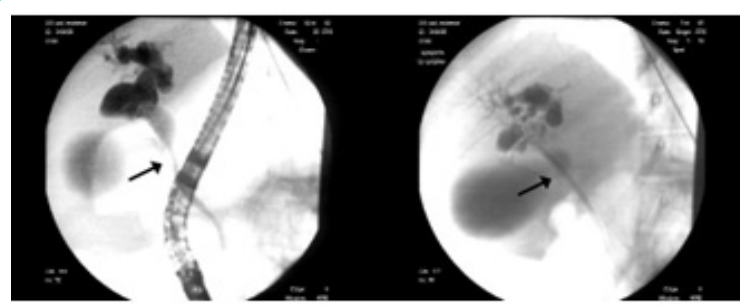

Figure 4: ERCP images. 4A: There is a stricture in the distal CHD and dilatation in the proximal CHD. 4B: ERCP image obtained after CBD stent placement.

\section{Discussion}

The first case of bile leakage (a patient who was kicked by a horse) was reported by Whipple in 1898 [5].The term "biloma" was coined by Gould and Patel in 1979. These authors reported a patient with extrahepatic bile leakage who had a history of upper abdominal trauma from a fight [6]. Spontaneous biloma is extremely rare, and choledocholithiasis and cholangiocarcinoma are reported as etiologic factors in most of these cases [3]. Rare causes have been reported, such as primary pancreatic malignancy [1], sickle cell disease $[7,8]$ and gallbladder tuberculosis [9]. We emphasized that the spontaneous biloma was related with sclerosing cholangitis because there was no risk factor for developing a biloma in this case, including trauma, gallstone, abdominal intervention from biopsy or surgery, or malignancy in the $\mathrm{CBD}$ and/or periampullary region. It was not found any further reports about spontaneous biloma-related sclerosing cholangitis in the literature.

The mechanism of spontaneous biloma formation is still unclear. One currently suggested pathogenic mechanism is increased intraductal pressure due to obstructive lesions or infarctions of the bile ducts. Trivedi et al. reported the first patient with spontaneous biloma, which occurred secondary to pancreatic malignancy, in 2009 [1]. They commented that an acute elevation in biliary pressure is unusual because of the relatively slow onset of ductal obstruction that occurs with pancreatic neoplasms [1]. Middleton et al. reported that spontaneous biloma occurred as a possible sequel of hepatic infarction in a patient with sickle cell disease [8]. Our case had benign strictures in the CBD, however there was no cholestasis upon her first admission and cholestasis could not caused by medical treatment for sclerosing cholangitis in our opinion. So, we suggested that the development of spontaneous biloma was related with inflammation of the intrahepatic biliary ducts due to sclerosing cholangitis rather than to benign strictures. It may have caused the increased pressure in $\mathrm{CBD}$, however we could not determine the source of the bile leak and the level of the increased biliary tree pressure.
Gallstones or extrahepatic biliary ductal compression, that was caused by a biloma must be kept in mind in the presence of increased levels of serum ALP, GGT, and total and direct bilirubin [10]. Antoine et al. reported the first postoperative biloma with extrinsic compression and obstruction of the CBD. Their case was the first with extrahepatic biliary compression secondary to biloma [10]. In our case, cholestasis developed on the tenth day of hospitalization (total bilirubin $5.7 \mathrm{mg} /$ dl, GGT $398 \mathrm{U} / \mathrm{L}$ ), which was attributed to the extrinsic compression of the CBD by a huge biloma. It was confirmed that the proximal portion of the CBD could not be displayed, and there were no stones in the gallbladder and/or biliary tree on ultrasonography and MRCP images.

The clinical findings of spontaneous biloma usually consist of abdominal pain, distension, anorexia, nausea, chills and fever. The leucocyte count, ESR and CRP values may increase in the presence of concomitant cholangitis. The necessary imaging methods for the diagnosis of the biloma are ultrasonography, computed tomography, MRI and MRCP. ERCP can be used for both diagnostic and therapeutic purposes [11]. Our case presented with abdominal distention and pain, but her biloma was misdiagnosed as massive ascites on the first abdominal ultrasonography. The diagnosis of biloma was confirmed by subsequent abdominal ultrasonography, MRI and needle aspiration.

Percutaneous and endoscopic interventions are appropriate therapeutic approaches, and these methods are preferred over surgery as the first step in treatment $[3,12,13]$. Surgery should remain the last option because it is associated with numerous complications [14]. In our case, percutaneous catheter drainage was performed initiallybecause of the serious abdominal pain by capsular stretching. Thereafter, we performed an ERCP to reduce the intraductal pressure and to prevent the recurrence of biloma and cholestasis. ERCP showed the compression of CBD by a huge biloma. The bile drainage ceased and the cholestasis resolved completely after endoscopic sphincterotomy and placement of CBD stent. Three months later, the patient had no complaints, and no sign of biloma was seen on MRI.

\section{Conclusion}

To our best knowledge, this is the first report of spontaneous biloma associated with sclerosing cholangitis in the literature. We suggested that the spontaneous biloma was related with inflammation ofthe intrahepatic biliary ducts due to sclerosing cholangitis, rather than to benign strictures that was causing the increased pressure in the CBD. So as this may not always be accompanied by cholestasis initially. Percutaneous drainage and/or endoscopic biliary decompression are curable and precise therapeutic approaches.

\author{
Abbreviations \\ MRI: Magnetic resonance imaging \\ MRCP: Magnetic resonance cholangiopancreatography \\ CHD: Common hepatic duct \\ IBD: Intrahepatic bile ducts \\ CA 19-9: serum carbohydrate antigen 19-9 \\ CEA: carcinoembryonic antigen \\ ERCP: Endoscopic retrograde cholangio-pancreatography \\ CBD: Common bile duct \\ TPN: Total parenteral nutrition
}

\section{Competing Interests}

The authors declare that they have no competing interests. 
Citation: Yucel A, Albayrak HM, Gumus M, Asil M, Ozbek O,A, et al. (2016) An Unexpected Complication in a Pediatric Patient With Sclerosing Cholangitis: A Huge Spontaneous Biloma. Int J Pediatr Neonat Care 2: 116. doi: https://doi.org/10.15344/2455-2364/2016/116

Page 4 of 5

\section{References}

1. Trivedi PJ, Gupta P, Phillips-Hughes J, Ellis A (2009) Biloma: An unusual complication in a patient with pancreatic cancer. World J Gastroenterol 15: 5218-5220.

2. Urbain D, Muls V, Kiromera A, Jeanmart J, Janne P, et al. (1992) Nontraumatic intrahepatic rupture of the biliary tree with biloma: The place of ERCP. Gastrointest Endosc 38: 379-381.

3. Fujiwara $\mathrm{H}$, Yamamoto $\mathrm{M}$, Takahashi $\mathrm{M}$, Ishida $\mathrm{H}$, Ohashi $\mathrm{O}$, et al (1998) Spontaneous rupture of an intrahepatic bile duct with biloma treated by percutaneous drainage and endoscopic sphincterotomy. Am J Gastroenterol 93: 2282-2284.

4. Feuerstein JD, Tapper EB (2013) Primary sclerosing cholangitis: An update. OA Hepatology1: 6.

5. Whipple C (1898) A case of traumatic rupture of the liver: formation of cystic swelling containing bile stained fluid. Lancet 1: 719 .

6. Gould L, Patel A (1979) Ultrasound detection of extrahepatic encapsulated bile: "biloma”. AJR Am J Roentgenol 132: 1014-1015.

7. Lebensburger J, Esbenshade A, Blakely M, Hankins J, Wang W (2008) Biloma and pneumobilia in sickle cell disease. Pediatr Blood Cancer 51: 288-290

8. Middleton JP, Wolper JC (1984) Hepatic biloma complicating sickle cell disease. A case report and a review of the literature. Gastroenterology 86 743-744.

9. Hahn ST, Park SH, Shin WS, Kim CY, Shinn KS (1995) Gallbladder tuberculosis with perforation and intrahepatic biloma. J Clin Gastroenterol 20: 84-86.

10. Mansour AY, Stabile BE (2000) Extrahepatic biliary obstruction due to postlaparoscopic cholecystectomy biloma. JSLS 4: 167-71.

11. Bas G, Okan I, Sahin M, Eryılmaz R, Isık A (2011) Spontaneous biloma managed with endoscopic retrograde cholangiopancreatography and percutaneous drainage: a case report. J Med Case Rep 5: 3.

12. Akhtar MA, Bandyopadhyay D, Montgomery HD, Mahomed A (2007) Spontaneous idiopathic subcapsularbiloma. J Hepatobiliary Pancreat Surg 14: $579-581$.

13. Kuligowska E, Schlesinger A, Miller KB, Lee VW, Grosso D (1983) Bilomas: a new approach to the diagnosis and treatment. Gastrointest Radiol 8: 237243.

14. Mizuno O, Kawamoto H, Fukatsu H, Harada R, Tsutsumi K, et al. (2008) An iatrogenic hepatic subcapsularbiloma successfully treated by percutaneous drainage and endoscopic biliary stenting. Endoscopy 40: 42-43. 